\title{
Composição química e isótopos de oxigênio em cassiterita e wolframita nos greisens do albita granito Palanqueta, depósito de estanho de Bom Futuro (RO)
}

\author{
Valmir da Silva Souza $a^{1}$ \& Nilson Francisquini Botelho ${ }^{1}$
}

\begin{abstract}
Resumo O Estado de Rondônia hospeda suítes graníticas meso a neoproterozóicas portadores de Sn, W, $\mathrm{Nb}, \mathrm{Ta}, \mathrm{F}$ e gemas, associadas à greisens, veios, brechas e pegmatitos, cujas informações sobre as composições químicas, isotópicas e de inclusões fluidas dos minerais-minérios ainda são discretas. Esse trabalho apresenta estudo sobre a composição química e isotópica $\left(\delta^{18} \mathrm{O}\right)$ de cassiterita e wolframita, encontradas nos greisens do albita granito Palanqueta, o qual faz parte do depósito de estanho do Bom Futuro. Nesses greisens os cristais de cassiterita exibem seu característico zonamento, ressaltado por faixas ricas em $\mathrm{Sn}$ alternadas por faixas ricas em Fe, Ti, Ta, $\mathrm{Nb}$ e W. Por outro lado, os cristais de wolframita mostram-se ricos em Fe em relação ao Mn, com franca migração para o membro ferberita $\left(\mathrm{Fe}_{0,52-0.86}-\mathrm{Mn}_{0,10-0.38}\right) \mathrm{WO}_{4}$, com baixos conteúdos em Nb, Ta e Sn. Tanto cassiterita como wolframita hospedam micro-inclusões de nióbio-tantalatos, provavelmente produtos de exsolução durante o abaixamento da temperatura. Quanto à composição isotópica $\left(\delta^{18} \mathrm{O}\right)$, cassiterita $(1,8 \mathrm{e} 2,0)$, wolframita $(1,4$ e 1,6) e quartzo $(9,6)$ exibem valores discrepantes e indicativos de fracionamento isotópico durante a ascensão dos fluidos responsáveis pela mineralização. Isso porque o quartzo é um mineral pouco suscetível ao fracionamento isotópico, cujo registro ficou melhor diagnosticado em cassiterita e wolframita. A composição isotópica de cassiterita, wolframita e quartzo aplicada para cálculos geotermométricos, assim como para o cálculo indireto da composição isotópica $\left(\delta^{18} \mathrm{O}\right)$ do fluido hidrotermal "fóssil" coexistente, estimam que cassiterita e wolframita foram precipitados no intervalo de temperatura de $420^{\circ}$ a $460^{\circ} \mathrm{C}$ e a partir do mesmo fluido hidrotermal de derivação magmática.
\end{abstract}

Palavras-chave: cassiterita, wolframita, depósito de estanho do Bom Futuro, Rondônia.

\begin{abstract}
The chemical composition and oxygen isotopes studies in cassiterite and wolframite from greisens of the Palanqueta albite granite, Bom Futuro tin deposit, Rondonia, Brazil. The Rondônia Ste hosts Meso- to Neoproterozoic granitic suites associated with important Sn, W, Nb, Ta, F, topaz and beryl mineralization confined in greisens, veins, breccia pipes and pegmatites. However, in these deposits, the chemical and isotopic composition, as well as fluid inclusions data, is still discrete. We provide in this paper new data on chemical and oxygen isotope composition of cassiterite and wolframite from greisens of the Palanqueta albite granite, in the Bom Futuro tin deposit. In these greisens the cassiterite crystals are typically zoned, with nearly pure $\mathrm{SnO}_{2}$ light zones and high $\mathrm{Fe}, \mathrm{Ti}, \mathrm{Ta}, \mathrm{Nb}$ e W dark zones, while the wolframite crystals are relatively $\mathrm{Fe}-$ rich, indicative of the specimen ferberite $\left(\mathrm{Fe}_{0,52-0,86}-\mathrm{Mn}_{0,10-0,38}\right) \mathrm{WO}_{4}$, with low contents of $\mathrm{Nb}, \mathrm{Ta}$ and $\mathrm{Sn}$. Both cassiterite and wolframite host micro-inclusions of $\mathrm{Nb}$-Ta minerals, probably as exsolution product during the lowering of temperature. The oxygen isotope $\left(\delta^{18} \mathrm{O} \%\right.$ ) composition of cassiterite $(1.8-2.0)$, wolframite $(1.4-$ 1.6) and quartz (9.6) exhibits discrepant values, indicative of isotope fractionation during rise of the hydrothermal fluids responsible for mineralization. This is because quartz is less susceptible to isotopic fractionation, better observed in cassiterite and wolframite. The oxygen isotope composition of cassiterite, wolframite and quartz to geotermometry, as well as to calculation of oxygen isotope composition in coexisten "fossil" hydrothermal fluids, suggests that cassiterite and wolframite were precipitated at the temperature range of $460^{\circ}$ to $420^{\circ} \mathrm{C}$ from the same hydrothermal fluid of magmatic derivation.
\end{abstract}

Keywords: cassiterite, wolframite, Bom Futuro tin deposit, Rondônia.

INTRODUÇÃ̃O O Estado de Rondônia hospeda, principalmente em suas regiões centro e centro-norte, suítes graníticas do tipo rapakivi com características geoquímicas de magmatismo do tipo A e intra-placa, alojadas durante sucessivos episódios magmáticos ocorridos entre 1606 e 974 Ma (Priem et al. 1971 e 1989, Waghorn 1974, Isotta et al. 1978, Bettencourt et al. 1999). Tais suítes graníticas formam a Província
Estanífera de Rondônia, cujas concentrações metálicas mais expressivas ( $\mathrm{Sn}, \mathrm{W}, \mathrm{Nb}, \mathrm{Ta}, \mathrm{F}$ e gemas) estão associadas aos três últimos episódios magmáticos, ocorridos entre 1314 e 974 Ma e representados pelas unidades regionais conhecidas por Suíte Intrusiva São Lourenço-Caripunas (1314-1309 Ma), Suíte Intrusiva Santa Clara (1082-1074 Ma) e Young Granites de Rondônia (995-991 Ma; Kloosterman 1968, Priem et 
al. 1971, Bettencourt et al. 1999 e 2005). Em geral, essas suítes graníticas são polifásicas, alojadas em níveis crustais rasos e a mineralização está associada a greisens, veios, pipes brechados e corpos pegmatíticos. Contudo, ainda são discretas as investigações sobre os mineraisminérios encontrados nesses depósitos, principalmente quanto à composição química, possíveis intercrescimentos ou exsoluções, isótopos estáveis e inclusões fluidas (Leite Jr. 2002, Sparrenberger 2003, Souza 2003, Bettencourt et al. 2005).

Este trabalho apresenta um estudo sobre a composição química e de isótopos de oxigênio $\left(\delta^{18} \mathrm{O}\right)$ em cassiterita e wolframita encontrados nos greisens do albita granito Palanqueta, depósito do Bom Futuro. São caracterizadas as substituiçõesiônicas, feições de zonação e intercrescimento, temperatura de formação e a natureza dos fluidos responsáveis pela mineralização, contribuindo assim com o entendimento dos processos metalogenéticos ocorridos na Província Estanífera de Rondônia.

PROCEDIMENTO ANALÍTICO As composições químicas dos cristais de cassiterita e wolframita foram obtidas através de análises em seções delgadas polidas desenvolvidas junto ao laboratório de microssonda eletrônica do Instituto de Geociências da Universidade de Brasília, utilizando equipamento modelo CAMECA SX50. As condições aplicadas para cada análise pontual nos cristais foram de uma tensão de aceleração de $20 \mathrm{kv}$, corrente de $40 \mathrm{nA}$ e exposição total de $15 \mathrm{seg}$.

Alguns cristais foram selecionados e submetidos à investigação através de microscópio eletrônico de varredura (MEV), modelo JEOL/JSM 5410, acoplado com EDS (Energy Dispersive Spectrometer) marca NORAN. Estas investigações foram realizadas no Departamento de Engenharia de Minas da Universidade Federal de Minas Gerais, cujo objetivo era obter imagens de elétrons retroespalhados (BSE), detalhando as feições internas dos minerais, tais como zonação, intercrescimento e sobrecrescimento, cujas observações são limitadas por meio da microscopia convencional.

As análises para isótopos de oxigênio $\left(\delta^{18} \mathrm{O}\right)$ foram realizadas em laboratório comercial da Scottish Universities Research \& Reactor Centre, na Escócia, aplicadas em quatro amostras puras de cassiterita, três de wolframita e quatro de quartzo. Cada amostra com cerca de $3 \mathrm{mg}$ foi submetida à diluição e subseqüente extração de seu conteúdo isotópico $\left(\delta^{18} \mathrm{O}\right)$, aplicado ao padrão internacional V-SMOW(\%o). A aplicação do quartzo nesse estudo objetivou a formação de pares isotópicos quartzo + cassiterita e quartzo + wolframita como geotermômetros e para o cálculo da composição isotópica $\delta^{18} \mathrm{O}$ do fluido hidrotermal.

CONTEXTO GEOLÓGICO O depósito de estanho do Bom Futuro, que é um dos principais representantes dos Young Granites de Rondônia, está localizado na porção centro-oeste de Rondônia, no município de Ariquemes, distante cerca de $200 \mathrm{~km}$ ao sul da capital Porto Velho (Fig. 1). Esse depósito de estanho é formado por um sistema do tipo vulcano-plutônico, encaixa- do em rochas paleoproterozóicas do Complexo Jamari (gnaisses, anfibolitos e xistos) e circundado por coberturas sedimentares aluviais holocênicas. O sistema vulcânico Bom Futuro ocupa a área centro-sul do depósito, local onde ocorrem brechas, diques de albita granito, riolito, traquito e corpos pegmatíticos, com a cassiterita associada a um sistema anelar de veios de quartzo-topázio. Já o sistema plutônico, denominado de Palanqueta é objeto deste estudo, está melhor representado a cerca de $500 \mathrm{~m}$ a nordeste do sistema vulcânico Bom Futuro, onde aflora um stock de biotita granito intrudido por um plug de albita granito, contendo zonas de greisens com cassiterita e wolframita (Villanova \& Franke 1995, Silva et al. 1995 e 1997, Souza \& Botelho 2002, Souza 2003).

No sistema plutônico Palanqueta o tipo petrográfico dominante é o biotita granito equigranular, o qual varia localmente para um biotita granito fino a porfirítico, enquanto o tipo petrográfico albita granito apresenta formato aproximadamente circular, cujos contatos são, em geral, ocultados por zonas de greisens e planos de falhas normais (Fig. 1). Os cristais de cassiterita e wolframita são encontrados em bolsões de greisens, associados principalmente aos tipos quartzo-topázio greisen e quartzo-topázio-zinnwaldita greisen, distribuídos nas porções nordeste e leste da zona de contato entre albita granito e biotita granito equigranular (Souza 2003, Souza et al. 2005) Entretanto, devido o conteúdo de cassiterita ser muito superior ao de wolframita, a presença de wolframita no depósito de Bom Futuro é considerada apenas uma ocorrência mineral importante no estudo de isótopos estáveis $\left(\delta^{18} \mathrm{O}\right)$, juntamente com cassiterita $\mathrm{e}$ quartzo, aplicados como geotermômetros para os greisens do sistema plutônico Palanqueta.

QUÍMICA MINERAL Os greisens apresentam arranjo microtextural heterogranular, com os cristais subédricos a euédricos de cassiterita e wolframita distribuídos de modo disseminado e/ou formando pequenos agregados junto à trama de minerais quartzo-topázio-zinnwaldita, normalmente associados a fluorita, esfalerita, pirita, calcopirita, galena, calcocita-digenita, hematita e monazita.

Cassiterita Os cristais de cassiterita são pouco fraturados e se destacam por seu zonamento comum, caracterizado pela alternância de faixas de cores castanha claro, laranja, vermelho, castanho escuro, marrom e marrom escuro (Fig. 2). As análises química revelaram que as faixas, cujas cores variam de castanho claro a laranja, apresentam conteúdos maiores em $\mathrm{SnO}_{2}$ e menores em $\mathrm{FeO}_{\text {(total) }}, \mathrm{TiO}_{2}, \mathrm{Ta}_{2} \mathrm{O}_{5}, \mathrm{Nb}_{2} \mathrm{O}_{5}$ e $\mathrm{WO}_{3}$, inversamente proporcional à composição química das zonas cujas cores variam de vermelho a marrom escuro (Tab. 1 e Fig. 2). Este comportamento está de acordo com os resultados conhecidos sobre a composição química de cristais zonados de cassiterita em outros depósitos de estanho, onde as faixas de cores mais claras são também mais puras em $\mathrm{SnO}_{2}$ (Giuliani 1987, Neiva 1996, Murciego et al. 1997, Costi et al. 2000).

Os principais mecanismos responsáveis por variações composicionais na cassiterita podem ser ex- 


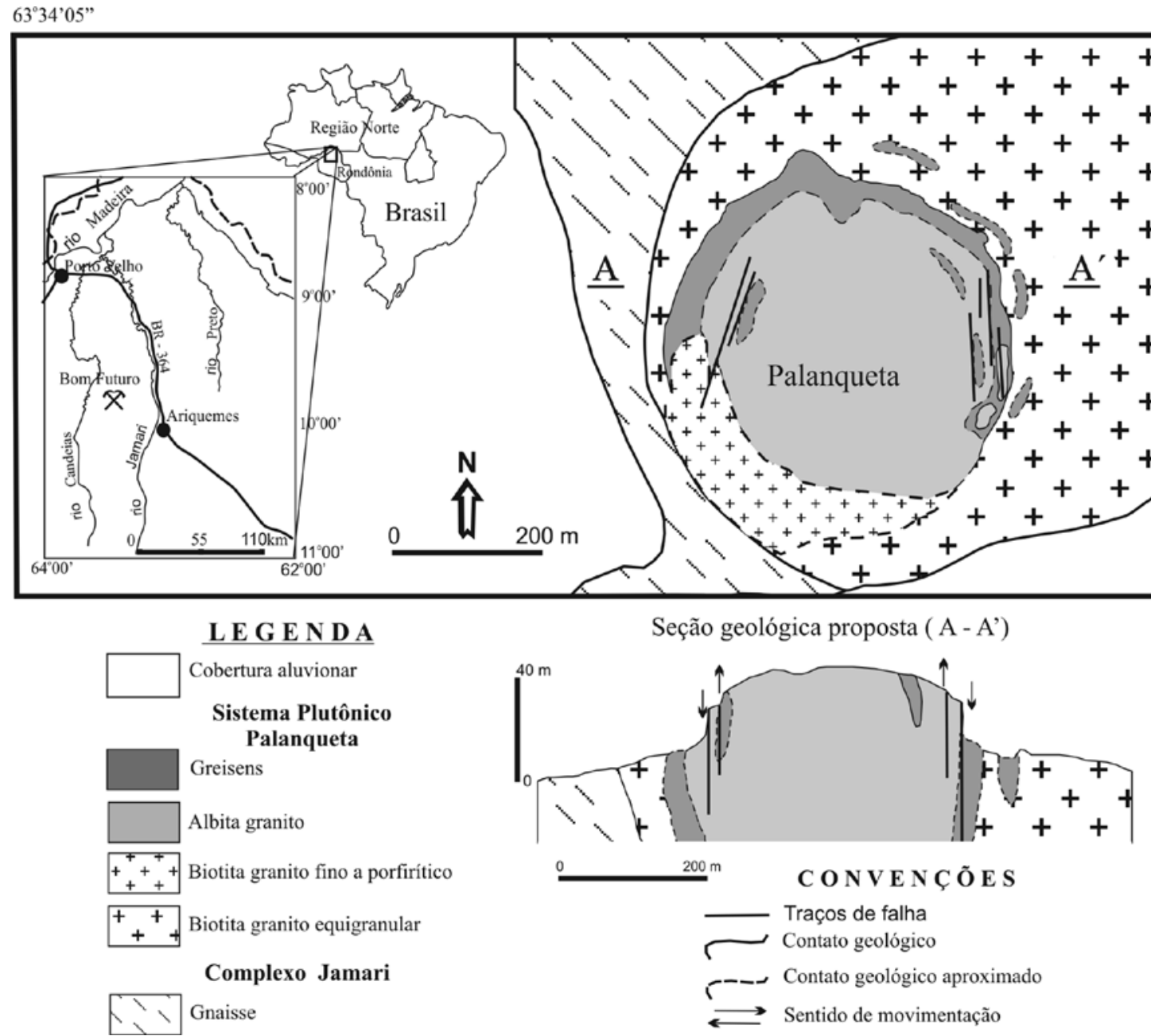

Figura 1 - Mapa geológico simplificado do sistema plutônico Palanqueta, contendo a proposta de uma seção geológica A-A' (modificado de Souza et al. 2005).

pressos pelas equações $2 \mathrm{Sn}^{4+}+\mathrm{O}^{2+} \leftrightarrow \mathrm{Ti}^{4+}+\mathrm{Fe}^{3+}+$ $\mathrm{OH}^{-}$e $3(\mathrm{Sn}, \mathrm{Ti})^{4+} \leftrightarrow 2(\mathrm{Nb}, \mathrm{Ta})^{5+}+\mathrm{Fe}^{2+}($ Neiva 1996 , Murciego et al. 1997, Möller et al. 1998), onde o Fe é a principal impureza na estrutura da cassiterita e o Ti participa durante a substituição do Sn pelo Fe. Por outro lado, o conteúdo de $\mathrm{WO}_{3}$ também tende a variar diretamente proporcional ao conteúdo de $\mathrm{FeO}_{\text {(total }}$, possibilitando que o $\mathrm{W}$ também participe da substituição entre $\mathrm{Sn}$ e $\mathrm{Fe}$, formando uma wolframita molecular, cujo mecanismo é expresso pela equação $2(\mathrm{Sn}, \mathrm{Ti})^{4+}$ $\leftrightarrow \mathrm{W}^{6+}+\mathrm{Fe}^{2+}$. Já o $\mathrm{Nb}$ e Ta entram como impurezas, principalmente nas zonas de cores mais escuras, através de substituições acopladas (Möller et al. 1988, Murciego et al. 1997). Tais substituições são observadas nos diagramas de correlação atômica entre $\mathrm{Sn} \times \mathrm{Fe}+\mathrm{Ti}$, $\mathrm{Fe}+\mathrm{Ta}+\mathrm{Nb}+\mathrm{W}$ e $\mathrm{Ti}+\mathrm{Ta}+\mathrm{Nb}+\mathrm{W}$ (Fig. 3), principalmente entre $\mathrm{Sn} \times \mathrm{Fe}+\mathrm{Ti}$, os quais mostram a formação de um trend decrescente, onde o aumento no conteúdo de Sn é acompanhado da diminuição nos conteúdos de $\mathrm{Fe}+\mathrm{Ti}+\mathrm{Nb}+\mathrm{Ta}+\mathrm{W}$.

Em geral, os cristais de cassiterita apresentam baixos conteúdos de $\mathrm{FeO}_{\text {(total) }}$ e, conseqüentemente, de $\mathrm{TiO}_{2}, \mathrm{Nb}_{2} \mathrm{O}_{5}, \mathrm{Ta}_{2} \mathrm{O}_{5}$ e $\mathrm{WO}_{3}$. Entretanto, nas análises sobre algumas faixas de cores castanho claro a laranja obser- vou-se, ocasionalmente, elevados conteúdos anômalos de $\mathrm{WO}_{3}$ e de $\mathrm{Nb}_{2} \mathrm{O}_{5}-\mathrm{Ta}_{2} \mathrm{O}_{5}$, os quais são atribuídos às micro-inclusões de cristais de wolframita e/ou exsoluções de nióbio-tantalatos diagnosticadas apenas por meio de EDS durante as análises por MEV (Fig. 4A). Tais exsoluções são consideradas produtos do abaixamento da temperatura e da mudança no estado de oxidação do $\mathrm{Fe}$ (Möller et al. 1988, Murciego et al. 1997, Neiva 1996). Freqüentemente, observa-se ainda que as microfraturas da cassiterita mostram-se preenchidas, principalmente, por pirita e esfalerita, demonstrando que a paragênese mineral sulfetada presente nos greisens foi precipitada posteriormente à cassiterita (Fig. 4B) .

Wolframita Os cristais de wolframita são opacos e de hábito tabular com comprimento entre de 1 a $3 \mathrm{~mm}$ (Fig. 5A). A wolframita é formada por uma solução sólida completa, cujos membros finais são representados por ferberita $\left(\mathrm{FeWO}_{4}\right)$ e hübnerita $\left(\mathrm{MnWO}_{4}\right)$, podendo sua fórmula estrutural ser expressa por $\mathrm{AWO}_{4}$, onde $\mathrm{A}=$ Fe,Mn (Hsu 1976, Waychunas 1991, Macavei \& Schulz 1993). As análises sobre os cristais de wolframita revelam franca tendência ao membro ferberita, cujo conteúdo de $\mathrm{FeO}_{\text {(total) }}$ é, em média, cerca de quatro vezes maior 



Figura 2 - Seqüencia de análises pontuais por microssonda eletrônica em cristais de cassiterita zonados mostrando as variações na composição química para óxidos em \% em peso. 
Tabela 1 - Dados das análises por microssonda eletrônica em cristais de cassiterita e wolframita. Composições dos óxidos em \% em peso e dos elementos em átomos por fórmula unitária, calculada na base de 2 oxigênios para cassiterita e na base de 4 oxigênios para wolframita. O símbolo (-) representa valores abaixo do limite de detecção.

\begin{tabular}{|c|c|c|c|c|c|c|c|c|c|c|c|c|c|c|c|c|c|c|c|c|c|c|c|c|c|}
\hline \multicolumn{26}{|l|}{ ss } \\
\hline & 1 & 2 & 3 & 4 & 5 & 6 & 7 & 8 & 9 & 10 & 11 & 12 & 13 & 14 & 15 & 16 & 17 & 18 & 19 & 20 & 21 & 22 & 23 & 24 & 25 \\
\hline & & & & & & & & & & & & & & & & & & & & & & & & & \\
\hline  &, 19 & 99,21 & 96,579 & 98,97 & 97,88 & 98,77 & 98,24 & 97,94 & 98,91 & 98,89 & $97,23,9$ & 96,77 & 99,06 & 97,62 & \begin{tabular}{|l|l|}
99,78 \\
\end{tabular} & 98,99 & 99,21 & 99,31 & 99,1 & 9,8 & 97,33 & 98,5 & 98,13 & 97,9 & 9 \\
\hline $\mathrm{iO}_{2}$ & \begin{tabular}{|l|}
0,57 \\
\end{tabular} & 1,19 & \begin{tabular}{|l|}
1,51 \\
\end{tabular} & 57 & 1,31 & 0,92 & 0,83 & 1,12 & \begin{tabular}{|l|l|}
0,91 \\
\end{tabular} & \begin{tabular}{|l|}
1,19 \\
\end{tabular} & \begin{tabular}{|l|}
1,66 \\
\end{tabular} & 1,83 & 0,72 & 1,07 & 0,55 & 0,79 & 0,86 & 0,75 & \begin{tabular}{|l|}
0,58 \\
\end{tabular} & 1,11 & 1,65 & 06 & 0,81 & 1,38 & 0.7 \\
\hline $\mathrm{a}_{2} \mathrm{O}_{5}$ & 22 & - & 0,05 & - & - & 0,07 & 0,02 & 0,31 & 0,22 & \begin{tabular}{|l|l|}
0,14 \\
\end{tabular} & 0,23 & - & - & 0,07 & 0,05 & 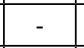 & - & 0,07 & 0,12 & 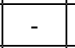 & 0,03 & - & \begin{tabular}{|l|}
0,08 \\
\end{tabular} & 05 & ,2 \\
\hline $\mathrm{Nb}_{2} \mathrm{O}_{5}$ & & - & - & - & - & - & - & 0,01 & 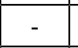 & - & - & 0,15 & - & - & - & - & - & - & - & - & 0,17 & - & - & 0,15 & 0,3 \\
\hline $\mathrm{VO}_{3}$ & 0,11 & - & 0,11 & - & - & - & - & - & 0,11 & \begin{tabular}{|l|}
0,07 \\
\end{tabular} & - & \begin{tabular}{|l|}
0,17 \\
\end{tabular} & 0,01 & 0,09 & - & - & 0,24 & - & - & \begin{tabular}{|l|}
0,02 \\
\end{tabular} & 0,1 & - & 0,1 & - & - \\
\hline $\mathrm{eO}$ & 1 & 0,04 & \begin{tabular}{l|l|}
51 &
\end{tabular} & 0,03 & 0,13 & 0,05 & 0,04 & 0,16 & \begin{tabular}{l|l}
05 &
\end{tabular} & \begin{tabular}{|l|}
0,07 \\
\end{tabular} & \begin{tabular}{|l|}
0,19 \\
\end{tabular} & 0,62 & 0,05 & 0,74 & 0,05 & 0,05 & 0,05 & \begin{tabular}{|l|}
0,03 \\
\end{tabular} & \begin{tabular}{|l|l|}
0,03 \\
\end{tabular} & \begin{tabular}{|l|}
0,38 \\
\end{tabular} & 0,09 & 0,05 & \begin{tabular}{|l|}
0,06 \\
\end{tabular} & \begin{tabular}{|l|}
0,15 \\
\end{tabular} & 0,3 \\
\hline otal & 99,92 & 100,45 & 98,779 & 99,58 & 99,33 & 99,82 & 99,16 & 99,55 & 100,22 & 100,38 & 99,33 & 99,55 & 99,86 & 99,62 & 100,44 & 99,84 & 100,38 & 100,18 & 99,84 & 99,32 & 99,38 & 9,62 & 99,11 & \begin{tabular}{|l|l|}
99,66 \\
\end{tabular} & 99,3 \\
\hline & 886 & 0,9760 & & & & & & & & & \begin{tabular}{|l|l|}
0,964 & 0
\end{tabular} & & & & 81 & & & & & & & & & & \\
\hline Ti & 0,011 & 0,022 c & 0,0280 & 0,011 & 0,025 & 0,017 & \begin{tabular}{|l|}
0,016 \\
\end{tabular} & 0,021 & \begin{tabular}{|l|}
0,017 \\
\end{tabular} & \begin{tabular}{|l|}
0,022 \\
\end{tabular} & \begin{tabular}{|l|l|}
0,031 & 0
\end{tabular} & \begin{tabular}{|l|}
0,034 \\
\end{tabular} & 0,014 & \begin{tabular}{|l|}
0,02 \\
\end{tabular} & 0,01 & 0,015 & \begin{tabular}{|l|}
0,016 \\
\end{tabular} & 0,014 & 0,011 & \begin{tabular}{|l|}
0,021 \\
\end{tabular} & 0,031 & \begin{tabular}{|l|}
0,02 \\
\end{tabular} & 0,015 & \begin{tabular}{|l|}
0,026 \\
\end{tabular} & 0 \\
\hline$\Gamma$ & - & - & - & - & - & 0,001 & - & 0,002 & \begin{tabular}{|l|l|}
0,002 \\
\end{tabular} & 0,001 & \begin{tabular}{|l|}
0,002 \\
\end{tabular} & - & - & 0,001 & - & - & - & - & 0,001 & - & - & - & 0,00 & - & ,0 \\
\hline $\mathrm{Nb}$ & - & - & - & - & - & - & - & - & - & - & - & \begin{tabular}{|l|l|}
0,002 \\
\end{tabular} & - & - & - & - & - & - & - & - & 0,00 & & & \begin{tabular}{|l|}
0,002 \\
\end{tabular} & 0 \\
\hline $\mathrm{W}$ & 001 & - & \begin{tabular}{|l|}
0,001 \\
\end{tabular} & - & - & - & - & - & \begin{tabular}{|l|l|}
0,001 \\
\end{tabular} & \begin{tabular}{|l|}
0,001 \\
\end{tabular} & - & \begin{tabular}{|l|}
0,002 \\
\end{tabular} & - & - & - & - & 0,002 & - & - & - & 0,001 & - & 0,00 & - & - \\
\hline $\mathrm{Fe}$ & - & 0010 & \begin{tabular}{|l|l|l|l|l|} 
& 0 \\
\end{tabular} & 0,001 & 0,003 & 0,001 & 0,001 & 0,003 & \begin{tabular}{|l|l|}
0,001 \\
\end{tabular} & \begin{tabular}{|l|}
0,002 \\
\end{tabular} & $\begin{array}{lll}04 & 0\end{array}$ & 0,013 & 0,001 & 0,016 & & 1 & & 0,001 & 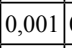 & 0 & 0,002 & 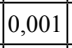 & 0 & 003 & \\
\hline Total & 998 & 1 & 1,055 & 1 & 1 & 0,999 & 0,999 & \begin{tabular}{|l|}
0,999 \\
\end{tabular} & \begin{tabular}{|c|}
0,998 \\
\end{tabular} & 1 & 1 & 1,005 & 0,999 & 1,006 & \begin{tabular}{|l|}
0,998 \\
\end{tabular} & 1 & 0,998 & (0,999 & 0,999 & 1,002 & 0,999 & 0,999 & 1 & 1 & 0,99 \\
\hline \multicolumn{26}{|l|}{ wolfi } \\
\hline & 1 & 2 & 3 & 4 & 5 & 6 & 7 & 8 & 9 & 10 & 11 & 12 & 13 & 14 & 15 & 16 & 17 & 18 & 19 & 20 & 21 & 22 & 23 & 24 & 25 \\
\hline $\mathrm{NO}_{3}$ & $|73,35|$ & 73,597 & \begin{tabular}{|l|l|}
73,97 &
\end{tabular} & 74,44 & 74,07 & 74,03 & 73,45 & 74,59 & 73,45 & 8 & $|73,23| 7$ & 7 & 73,74 & 74,76 & 74 & $|73,27|$ & \begin{tabular}{|l|l|}
74,28 \\
\end{tabular} & 74,18 & $\mid 74,71$ & $|75,93| 7$ & 75,31 & $\mid 76,39$ & $|76,28|$ & $|75,69|$ & 15 \\
\hline $\mathrm{b}_{2} \mathrm{O}_{5}$ & 1,66 & ,66 & 1,52 & 53 & 1,25 & 1,38 & 0,26 & 1,16 & 53 & 43 & 1,42 & 1,55 & \begin{tabular}{|l|}
1,84 \\
\end{tabular} & 0,75 & 1,2 & 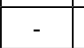 & \begin{tabular}{|l|}
- \\
\end{tabular} & \begin{tabular}{|l|}
- \\
\end{tabular} & \begin{tabular}{|l|}
- \\
\end{tabular} & \begin{tabular}{|l|}
- \\
\end{tabular} & - & \begin{tabular}{|l|}
- \\
\end{tabular} & \begin{tabular}{|l|}
- \\
\end{tabular} & - & -1 \\
\hline $\mathrm{a}_{2} \mathrm{O}_{5}$ & 65 & 0,61 & 0,5 & 0,75 & 0,48 & 0,55 & 0,1 & 0,38 & 0,61 & 0,37 & 0,5 & 0,63 & 0,37 & 0,06 & 0,59 & 1,03 & 0,35 & 0,06 & \begin{tabular}{|l|}
0,51 \\
\end{tabular} & \begin{tabular}{|l|l|}
0,23 \\
\end{tabular} & 0,18 & - & - & 0,03 & \\
\hline $\mathrm{riO}_{2}$ & 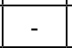 & - & - & 0,01 & - & - & \begin{tabular}{|l|}
0,04 \\
\end{tabular} & - & - & 0,03 & \begin{tabular}{|l|l|}
0,02 \\
\end{tabular} & 0,01 & - & 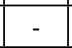 & - & - & - & - & - & 0,04 & 0,26 & - & 0,03 & 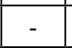 & - \\
\hline $\mathrm{nO}_{2}$ & 12 & 0,07 & \begin{tabular}{l|l|}
0,09 \\
\end{tabular} & 8 & 0,12 & 0,09 & - & \begin{tabular}{|l|}
0,08 \\
\end{tabular} & 18 & 6 & \begin{tabular}{|l|}
0,14 \\
\end{tabular} & 0,15 & \begin{tabular}{|l|}
0,09 \\
\end{tabular} & 0,08 & 0,09 & \begin{tabular}{|l|l|}
0,06 \\
\end{tabular} & 0,06 & 0,1 & \begin{tabular}{|l|}
0,08 \\
\end{tabular} & 0,02 & & \begin{tabular}{|l|}
- \\
\end{tabular} & - & 84 & 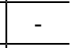 \\
\hline $\mathrm{eO}_{\text {tot }}$ &, 51 & \begin{tabular}{|l|l}
19,29 & 1 \\
\end{tabular} & \begin{tabular}{|l|l|}
19,39 & 1 \\
\end{tabular} & 13,47 & 19,12 & 19,37 & 12,36 & 19,76 & 18,26 & \begin{tabular}{|l|}
19,32 \\
\end{tabular} & \begin{tabular}{|l|l}
16,33 & 1 \\
\end{tabular} & 12,45 & 19,76 & 18,87 & 18,82 & \begin{tabular}{|l|}
19,39 \\
\end{tabular} & 19,76 & 19,58 & 19,5 & \begin{tabular}{|l|l|}
19,62 & 1 \\
\end{tabular} & 19,59 & 19,44 & 19,67 & 19,82 & 19,4 \\
\hline InO & & & \begin{tabular}{|l|l|}
3,84 \\
\end{tabular} & & 3,95 & 3,69 & & 3,46 & & & & 10,54 & 8 & 9 & & & & & & 7 & 4 & 2,31 & 2,35 & 37 & 2, \\
\hline tal & 9 & \begin{tabular}{|l|l|}
99,02 & 9
\end{tabular} & \begin{tabular}{|l|l}
99,349 \\
\end{tabular} & 99,36 & 99,01 & 99,14 & 96,08 & \begin{tabular}{|l|}
99,45 \\
\end{tabular} & \begin{tabular}{|l|}
98,94 \\
\end{tabular} &, 1 & \begin{tabular}{|l|l}
98,68 & 9
\end{tabular} & \begin{tabular}{|l|}
99,38 \\
\end{tabular} & 99,39 & 98,62 & 98,84 & 51 & 6,79 & 96,27 & \begin{tabular}{|l|}
97,12 \\
\end{tabular} & \begin{tabular}{|l|l}
98,23 &
\end{tabular} & 97,87 & 98,14 & 98,35 & \begin{tabular}{|l|}
97,97 \\
\end{tabular} & , \\
\hline & & & & & & & & & & & & & & & & & & & & & & & & & \\
\hline & 963 & \begin{tabular}{|l|l|l|}
0,966 & 0 \\
\end{tabular} & \begin{tabular}{|l|l|l|}
0,968 & 0 \\
\end{tabular} & 0,975 & 0,974 & 0,972 & 0,997 & 0,977 & 0,966 & 969 & $\begin{array}{lll}0,965 & 0 \\
\end{array}$ & \begin{tabular}{|l|l|}
0,969 \\
\end{tabular} & 0,964 & 0,989 & $0,9 / 6$ & 1,000 & 1,000 & 1,000 & 1,006 & \begin{tabular}{|l|l}
1,01 \\
\end{tabular} & 1,009 & 1,013 & 1,006 & 1,02 & 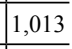 \\
\hline $\mathrm{Nb}$ & 038 & \begin{tabular}{|l|l|}
0,038 & 0
\end{tabular} & \begin{tabular}{|l|l|}
0,035 & 0 \\
\end{tabular} & 0,035 & 0,029 & 0,032 & 0,006 & 0,027 & 0,035 & 933 & \begin{tabular}{|l|l}
0,033 & 0 \\
\end{tabular} & 0,036 & 0,042 & 0,017 & \begin{tabular}{l|l}
0,028 \\
\end{tabular} & \begin{tabular}{|l|}
0,000 \\
\end{tabular} & 0,000 & 0,0000 & 0,000 & \begin{tabular}{|c|c|c|}
0,000 & \\
\end{tabular} & 0,000 & 0,000 & 0,000 & 0,000 & $t$ \\
\hline &, 009 & $0,008 \quad 0$ & 0,007 & 0,01 & 0,007 & 0,008 & 0,002 & 0,005 & 0,009 & 0,005 & $0,007 \quad 0$ & 0,0090 & 0,005 & 0,001 & 0,008 & \begin{tabular}{|l|l|}
0,020 \\
\end{tabular} & 0,005 & \begin{tabular}{|l|l|}
0,015 & 0 \\
\end{tabular} & 0,005 & 0,001 c & 0,007 & 0,003 & 0,00 & & 0 \\
\hline & & & \begin{tabular}{l|l}
- & 0 \\
\end{tabular} & \begin{tabular}{|l|}
0,001 \\
\end{tabular} & - & $-\quad$ & 0,002 & - & & \begin{tabular}{|l|l|}
0,001 \\
\end{tabular} & \begin{tabular}{|l|l|l|}
0,001 & 0
\end{tabular} & \begin{tabular}{|l|}
0,001 \\
\end{tabular} & | & & & 0,008 & & & & - & - & 0,002 & 0,01 & & , 0 \\
\hline$\pi$ & 003 & \begin{tabular}{l|l|l|l|l|l|l}
0,001 & 0
\end{tabular} & \begin{tabular}{|l|l|}
0,002 & 0 \\
\end{tabular} & 0,004 & 0,002 & \begin{tabular}{|l|l|}
0,002 \\
\end{tabular} & 0 & \begin{tabular}{|l|l|}
0,002 \\
\end{tabular} & \begin{tabular}{|l|l|}
0,004 \\
\end{tabular} & \begin{tabular}{|l|l|}
0,001 \\
\end{tabular} & \begin{tabular}{|l|l|}
0,003 & 0 \\
\end{tabular} & \begin{tabular}{|l|l|}
0,003 & 0 \\
\end{tabular} & \begin{tabular}{|l|}
0,002 \\
\end{tabular} & 0,002 & 0,002 & \begin{tabular}{l|l}
0,02 & \\
\end{tabular} & \begin{tabular}{|l|}
0,004 \\
\end{tabular} & \begin{tabular}{|l|l|}
0,001 & 0 \\
\end{tabular} & 0,001 & \begin{tabular}{|l|c|}
0,002 & \\
\end{tabular} & 0,002 & 0,001 & 0,002 & & \\
\hline $\mathrm{e}^{2+}$ & 0,793 & \begin{tabular}{|l|l|}
0,783 & 0 \\
\end{tabular} & \begin{tabular}{|l|l|l|l|l|l|}
0,787 & \\
\end{tabular} & 0,544 & 0,786 & 0,793 & 0,539 & 0,812 & \begin{tabular}{|l|}
0,741 \\
\end{tabular} & \begin{tabular}{|l|}
0,788 \\
\end{tabular} & \begin{tabular}{|l|l}
0,66 & 0 \\
\end{tabular} & \begin{tabular}{|l|l|l|l|l|l|l|}
0,45 & \\
\end{tabular} & \begin{tabular}{|l|}
0,797 \\
\end{tabular} & 0,795 & \begin{tabular}{l|}
0,777 \\
\end{tabular} & 0,850 & 0,860 & \begin{tabular}{|l|l|}
0,854 & 0 \\
\end{tabular} & 0,863 & \begin{tabular}{|l|}
0,86 \\
\end{tabular} & 0,85 & 0,845 & 0,845 & 0,838 &, 84 \\
\hline $\mathrm{e}^{3+}$ & 035 & \begin{tabular}{|c|c|}
0,034 & 0 \\
\end{tabular} & 20 & \begin{tabular}{|l|l|} 
\\
\end{tabular} & 0,026 & 0 & & & 34 & & & & & & & & & & & 0 & & & 0 & & 0 \\
\hline $\mathrm{Mn}$ & 154 & \begin{tabular}{|l|l|}
0,162 & 0 \\
\end{tabular} & 0,1640 & 0,383 & 0,17 & \begin{tabular}{|c|}
0,159 \\
\end{tabular} & 0,437 & 0,148 & \begin{tabular}{|l|}
0,21 \\
\end{tabular} & 0,175 & \begin{tabular}{|l|l|}
0,302 & 0 \\
\end{tabular} & \begin{tabular}{|l|l}
0,451 & 0 \\
\end{tabular} & \begin{tabular}{|l|}
0,153 \\
\end{tabular} & 0,177 & \begin{tabular}{l|l}
0,177 \\
\end{tabular} & \begin{tabular}{l|c}
0,099 \\
\end{tabular} & \begin{tabular}{|l|}
0,099 \\
\end{tabular} & \begin{tabular}{l|l}
0,105 & 0 \\
\end{tabular} & 0,103 & \begin{tabular}{|c|c|c|} 
\\
\end{tabular} & 0,102 & 0,103 & 0,105 & \begin{tabular}{|l|l|}
0,101 \\
\end{tabular} & {$[0,1$} \\
\hline Total & 997 & \begin{tabular}{|l|l|}
1,989 &
\end{tabular} & $1,995 \mid 1$ & $\mid 1,977$ & 1,994 & 1,989 & 1,986 & 1,994 & 1,999 & 2,003 & 2,006 & \begin{tabular}{|l|l|l|} 
& 1,995 \\
\end{tabular} & \begin{tabular}{|l|}
1,999 \\
\end{tabular} & 1,992 & 1,992 & \begin{tabular}{|l|l|}
1,997 \\
\end{tabular} & 1,968 & \begin{tabular}{|l|l}
1,975 & 1 \\
\end{tabular} & 1,978 & \begin{tabular}{|l|l|}
1,976 \\
\end{tabular} & 1,97 & 1,967 & 1,969 & 1,959 & \\
\hline & & & & & & & & & & & & & & & & & & & & & & & & & \\
\hline $\begin{array}{l}3+= \\
-W)\end{array}$ & ( & 0 & 0 & 0 & c & 0 & 0 & 3 ( & 4 & & 0 & 0 & 36 & 11 & & 0 & 0 & 0 & 0 & 0 & 0 & 0 & 0 & 0 & 0 \\
\hline $\mathrm{b}+\mathrm{Ta}$ & 0,047 & 0,0460 & \begin{tabular}{|l|l|}
0,042 & 0
\end{tabular} & 0,045 & 0,036 & \begin{tabular}{|l|}
0,04 \\
\end{tabular} & 0,008 & (0,032 & |0,044 & 0,038 & \begin{tabular}{l|l}
0,04 & 0
\end{tabular} & $|0,045| 0$ & & 0,018 & 0,036 & 0,02 & ,005 & 0,015 & 0,005 & $0,001 \mid \mathrm{C}$ & 0,00 & 0,003 & $0,003 \mid$ & 0 & 0 \\
\hline
\end{tabular}

que o conteúdo de $\mathrm{MnO}$ (Tab. 1 e Fig. 5B), com sua fórmula estrutural expressa por $\left(\mathrm{Fe}_{0,52-0.86}, \mathrm{Mn}_{0,10-0,38}\right) \mathrm{WO}_{4}$. No diagrama de correlação triangular para concentrações atômicas $\mathrm{W}$ x Fe x Mn, também se observa franca tendênciadas análises se deslocarem para o vértice do $\mathrm{Fe}$ (Fig. 5C).

Os cristais de wolframita também apresentam impurezas de $\mathrm{Nb}$, Ta e $\mathrm{Sn}$, as quais estão na ordem de elementos traços. O conteúdo de $\mathrm{Nb}_{2} \mathrm{O}_{5}$ é, em média, cerca de três vezes maior que o conteúdo de $\mathrm{Ta}_{2} \mathrm{O}_{5}$ (Fig.
5D), enquanto que o conteúdo de $\mathrm{SnO}_{2}$ é variável, porém bem inferior ao conteúdo de $\mathrm{Ta}_{2} \mathrm{O}_{5}$. A incorporação de $\mathrm{Nb}$ e Ta na estrutura da wolframita pode ser resultado de substituições acopladas, expressas pela equação $\mathrm{Fe}^{2+}$ $+\mathrm{W}^{6+} \leftrightarrow \mathrm{Fe}^{3+}+(\mathrm{Nb}, \mathrm{Ta})^{5+}$ (Polya 1988), a qual pode ser diagnosticada através do diagrama triangular de correlação atômica $\mathrm{Fe}^{+3} \times \mathrm{Nb}+\mathrm{Ta}$ (Fig. 5E). Essa substituição é confirmada pela deficiência em $\mathrm{W}^{6+}$ acompanhada do aumento de $\mathrm{Nb}^{5+}+\mathrm{Ta}^{5+}$ (Tab. 1). A deficiência de carga eletrostática decorrente dessa substituição deve 



Figura 3 - Diagramas de correlações atômicas (Sn $x$ $\mathrm{Fe}+\mathrm{Ti}, \mathrm{Fe}+\mathrm{Ta}+\mathrm{Nb}+W$ e $\mathrm{Ti}+\mathrm{Ta}+\mathrm{Nb}+W$ ) aplicados às cassiteritas de greisen do sistema plutônico Palanqueta.

ser então compensada pela oxidação de $\mathrm{Fe}^{2+}$ em $\mathrm{Fe}^{3+}$.

Por outro lado, investigações por meio da $\mathrm{MEV}$, aplicando EDS em wolframita, diagnosticaram a presença de diversas micro-inclusões ou exsoluções de nióbiotantalatos (Fig. 6A e 6B). Como o membro ferberita $\left(\mathrm{FeWO}_{4}\right)$ mostra-se estável sob condições oxidantes e temperaturas acima de $400^{\circ} \mathrm{C}$, podendo inclusive ocorrer associado com minerais ricos em Fe, tais como hemati-
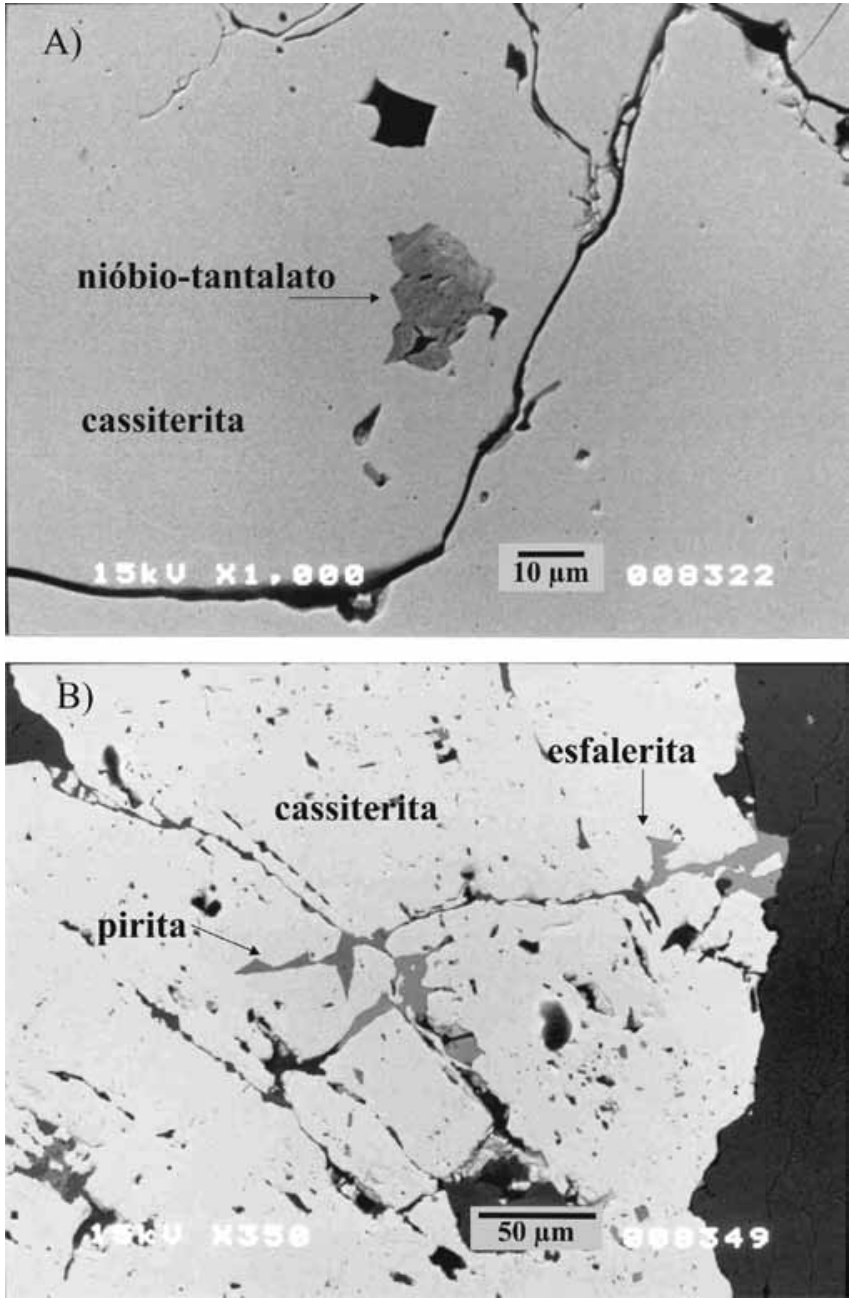

Figura 4 - Imagens elétrons retroespalhados (BSE) obtidas por meio de microscópio eletrônico em cristais de cassiterita, ressaltando exsoluções de nióbio-tantalato (A) e microfraturas preenchidas por pirita e esfalerita (B).

ta (Hsu 1976, Ivanova 1988), é provável que os nióbiotantalatos encontrados na wolframita sejam produtos de exsolução durante o abaixamento da temperatura.

ISÓTOPOS DE OXIGÊNIO $\left(\boldsymbol{\delta}^{18} \mathrm{O}\right)$ A composição isotópica de cassiterita, wolframita e quartzo expressa na tabela 2 revela valores comparativamente discrepantes, os quais podem ser indicativos de um processo de fracionamento isotópico durante a ascensão dos fluidos responsáveis pela mineralização. Como o quartzo é um mineral pouco suscetível ao fracionamento isotópico (Faure1986, Taylor Jr. 1978), tal registro ficou melhor diagnosticado em cassiterita e wolframita.

Para o estudo geotermométrico utilizando os pares quartzo+cassiterita e quartzo+wolframita, admitiu-se que a cristalização desses minerais ocorreu em equilíbrio isotópico. Nesse sentido, os cálculos para os intervalos de temperatura foram elaborados com base na curva de fracionamento regida pela equação 1000 $\ln \alpha=\mathrm{A} \times 10^{6} / \mathrm{T}^{2}+\mathrm{B} \times 10^{3} / \mathrm{T}+\mathrm{C}$ (Zhang et al. 1994), cujos parâmetros A, B e C são constantes numéricas de- 

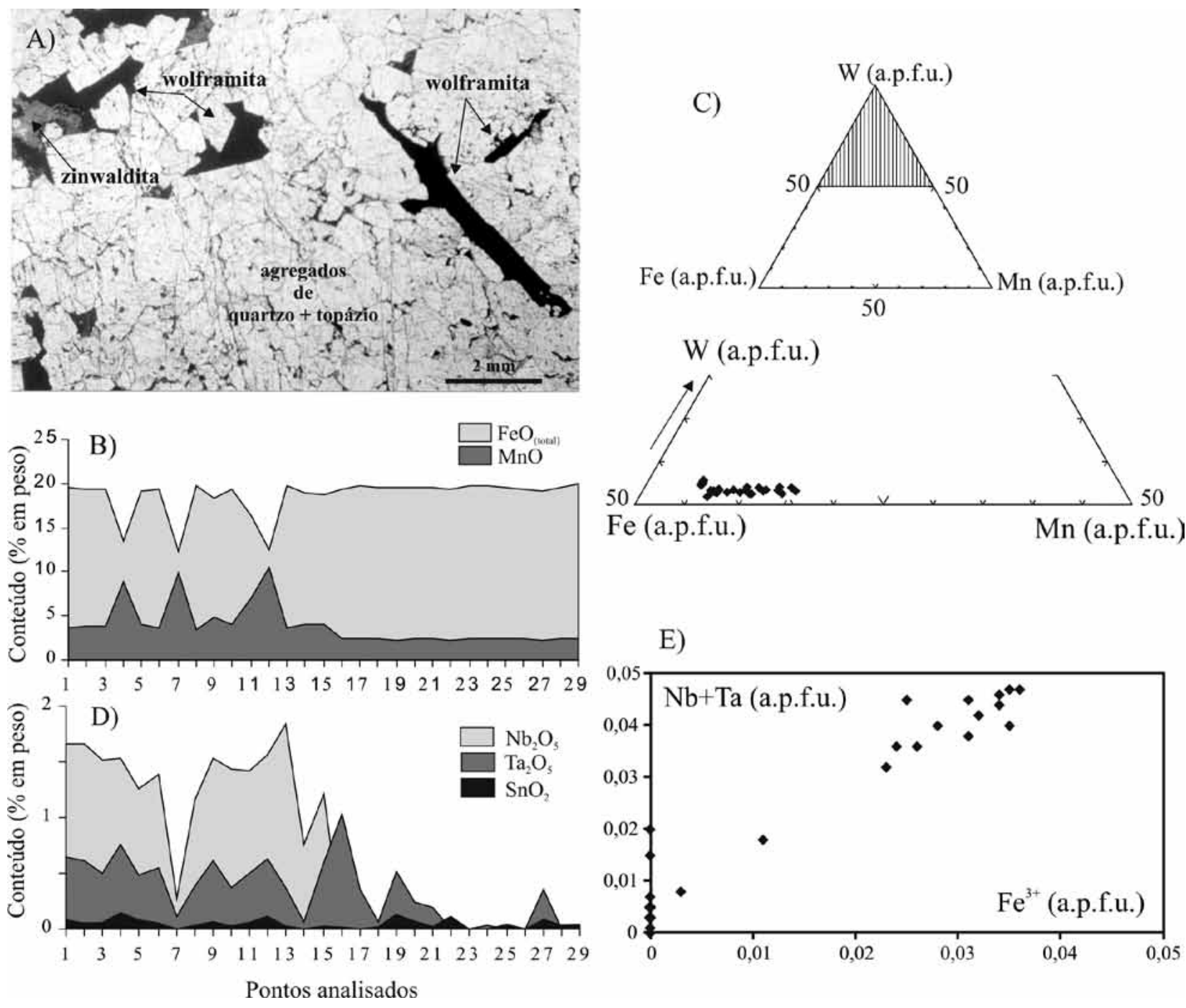

E)

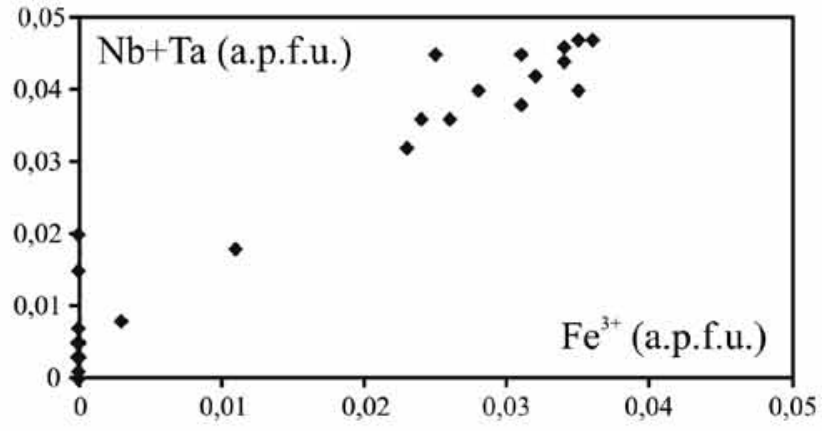

Figura 5 - A) fotomicrografia ressaltando a distribuição de cristais de wolfranita junto a trama quartzotopázio-zinwaldita greisens do sistema plutônico Palanqueta; B) seqüencia de análises pontuais por microssonda eletrônica em cristais de wolframita mostrando as variações nos conteúdos de $\mathrm{FeO}_{\text {total }}$ e MnO; C) diagrama de correlação atômica Fe-W-Mn aplicado aos cristais de wolframita; D) seqüencia de análises pontuais por microssonda eletrônica em cristais de wolframita mostrando as variações nos conteúdos de $\mathrm{Nb}_{2} \mathrm{O}_{5}, \mathrm{Ta}_{2} \mathrm{O}_{5}$ e $\mathrm{SnO}_{2}$. E) diagrama de correlação atômica $\mathrm{Fe}^{+3} \times \mathrm{Nb}+$ Ta aplicado aos cristais de wolframita;

terminadas experimentalmente por Zhang et al. (1994) para o sistema quartzo-cassiterita-água e quartzo-wolframita-água (Tab. 3). O valor de $\alpha$ foi obtido através da relação $\alpha=1000+\delta_{\text {mineral A }} / 1000+\delta_{\text {mineral B }}$ (Faure 1986; Rollinson 1993) e o valor de T é expresso em temperatura absoluta ou ${ }^{\circ} \mathrm{K}$.

$\mathrm{O}$ intervalo geotermométrico obtido através do par quartzo+cassiterita foi de $451^{\circ}$ a $462^{\circ} \mathrm{C}$, enquanto que no par quartzo+wolframita foi de $419^{\circ}$ a $433^{\circ} \mathrm{C}$ (Tab. 2). A utilização do par quartzo+wolframita apresenta uma margem de erro de cerca de $\pm 15^{\circ} \mathrm{C}$ para temperatura de equilíbrio isotópico acima de $300^{\circ} \mathrm{C}$, enquanto que as possíveis substituições de $\mathrm{Fe}$ e Mn entre os membros ferberita e hübnerita interferem de modo desprezível no fracionamento isotópico na wolframita (Zheng 1992, Zhang et al. 1994). Mesmo considerando esta margem de erro, o intervalo de temperatura isotó- pica obtido para o greisens mostra-se compatível com as informações do estudo de inclusões fluidas realizados em cristais de cassiterita por Souza (2003), no qual a temperatura mínima de homogeneização obtida nos fluidos foi entre 340 e $420^{\circ} \mathrm{C}$.

Para o cálculo indireto da composição isotópica $\delta^{18} \mathrm{O}$ "fóssil" do fluido hidrotermal responsável pela deposição de cassiterita e wolframita nos greisens, utilizou-se os valores calculados de temperatura isotópica através dos pares quartzo+cassiterita e quartzo+wolframita aplicados na mesma equação 1000 $\ln \alpha=\mathrm{A} \times 10^{6} / \mathrm{T}^{2}+\mathrm{B} \times 10^{3} / \mathrm{T}+\mathrm{C}$ (Zhang et al.1994). Neste cálculo, as constantes A, B e C utilizadas estão relacionadas ao par mineral+água determinados por Zhang et al. (1994) e expressos na tabela 3. O valor calculado da temperatura isotópica deve ser corrigido de ${ }^{\circ} \mathrm{C}$ para ${ }^{\circ} \mathrm{K}$, compondo a seguinte relação: 1000 



Figura 6 - Imagens elétrons retroespalhados (BSE) obtidas por meio de microscópio eletrônico em cristais de wolframita (A), ressaltando exsoluções de nióbiotantalato $(B)$.

$\ln \alpha=\delta^{18} \mathrm{O}_{\text {(mineral) }}-\delta^{18} \mathrm{O}_{(\mathrm{H} 2 \mathrm{O})}$, onde o valor de $\delta^{18} \mathrm{O}_{\text {(mineral }}$ corresponde à composição isotópica de cassiterita ou wolframita. Em relação ao quartzo, o cálculo indireto da composição isotópica $\delta^{18} \mathrm{O}$ para o fluido hidrotermal coexistente foi elaborado com base na curva de fracionamento quartzo-água, regida pela equação $1000 \ln \alpha=$ $3,38 \times 10^{6} / \mathrm{T}^{2}-3,40$ e proposta por Clayton et al. (1972) para um intervalo de temperatura entre $200^{\circ}$ e $500^{\circ} \mathrm{C}$. Para este cálculo, considerou-se a relação $\delta^{18} \mathrm{O}$ $-\delta^{18} \mathrm{O}_{(\mathrm{H} 2 \mathrm{O})}=1000 \ln \alpha$, admitindo-se o valor de 1000 $\ln \alpha$ para a temperatura de $500^{\circ} \mathrm{C}$, equivalente a $773^{\circ} \mathrm{K}$, considerada como próxima às temperaturas isotópicas obtidas através dos pares minerais quartzo+cassiterita $\mathrm{e}$ quartzo+wolframita.

A composição isotópica $\delta^{18} \mathrm{O}$ indireta do fluido hidrotermal coexistente com quartzo, cassiterita e wolframita apresenta um intervalo entre 4,26 e 7,43\%. Segundo Taylor Jr. (1974), fluidos de derivação magmática apresentam um típico intervalo isotópico $\delta^{18} \mathrm{O}$ entre 5,5 e 10,0\%o. Como a maioria das composições isotópicas calculadas está no intervalo de 5,75 e $7,43 \%$, o fluido hidrotermal responsável pela deposição de cassiterita pode ser considerado como predomi-
Tabela 2 - Valores calculados para os intervalos de temperaturas de cristalização dos minerais quartzo-cassiterita-wolframita e para o intervalo de composição isotópica do fluido hidrotermal coexistente $\left(\delta^{18} \mathrm{O}_{2} \mathrm{O}\right)$.

\begin{tabular}{c|c|c|c}
\hline Par mineral & $\begin{array}{c}\delta^{18} \mathrm{O} \text { SMOW } \\
(\%)\end{array}$ & $\begin{array}{c}\text { Temperatura } \\
\left({ }^{\circ} \mathrm{C}\right)\end{array}$ & $\begin{array}{c}\delta^{18} \mathrm{O} \mathrm{H}_{2} \mathrm{O} \\
(\%)\end{array}$ \\
\hline $\begin{array}{c}\text { Quartzo } \\
\text { Cassiterita }\end{array}$ & 9,6 & \multirow{2}{*}{451 e 462} & 7,33 \\
\cline { 2 - 2 } \cline { 4 - 4 } $\begin{array}{c}\text { Quartzo } \\
\text { Wolframita }\end{array}$ & 9,6 & 419 e 433 & 5,84 e 6,17 \\
\hline
\end{tabular}

Tabela 3 - Fracionamentos isotópicos do oxigênio em cassiterita e wolframita, cujos parâmetros $A, B$ e C foram utilizados para o cálculo da temperatura nos greisens do albita granito Palanqueta (Zhang et al. 1994).

\begin{tabular}{c|c|c|c|c|c|c|c}
\hline & \multicolumn{2}{|c|}{ quartzo-mineral } & \multicolumn{3}{c|}{ mineral-água } & \\
\hline Mineral & A & B & C & A & B & C & temperatura \\
\hline Cassiterita & $-6,87$ & 26,29 & $-15,49$ & 10,13 & $-26,09$ & 12,58 & $250^{\circ}-500^{\circ} \mathrm{C}$ \\
\hline Wolframita & $-1,04$ & 10,74 & $-6,27$ & 3,13 & $-6,42$ & $-0,12$ & $200^{\circ}-420^{\circ} \mathrm{C}$ \\
\hline
\end{tabular}

nantemente de origem magmática. As exceções são os fluidos hidrotermais com composições isotópicas $\delta^{18} \mathrm{O}$ abaixo de 5,5\%, determinadas em wolframita, cujo rebaixamento isotópico pode ser atribuído à interação do fluido hidrotermal magmático com fluido meteórico durante a greisenização. Como o quartzo é um mineral muito mais resistente a mudanças isotópicas (Taylor Jr. 1974, Faure1986, Taylor Jr. 1978), o registro da interação entre fluidos de diferentes origens ficou melhor diagnosticado na wolframita. Entretanto, não se descarta a possibilidade das exsoluções de nióbio-tantalatos identificadas na wolframita influenciarem, mesmo que de modo discreto, na sua composição isotópica.

CONSIDERAÇÕES FINAIS A associação cassiterita-wolframita é comum em sistemas hidrotermais de origem magmática, aprisionados em greisens e vinculados a granitos altamente fracionados ricos em voláteis. Entretanto, os conteúdos de cassiterita e wolframita podem variar em diferentes proporções $(\mathrm{Sn}>\mathrm{W}, \mathrm{Sn} \approx \mathrm{W}$ ou $\mathrm{Sn}<\mathrm{W}$ ), cujos mecanismos físico-químicos responsáveis por essas proporcionalidades ainda são motivos de discussão. Em geral, os pesquisadores consideram a atuação conjunta ou em parte dos seguintes mecanismos: a) variações químico-mineralógicas nos sistemas graníticos portadores de $\mathrm{Sn}$ ou $\mathrm{W}, \mathrm{b}$ ) diferenças nos coeficientes de partição entre Sn e W durante o fracionamento, c) diferenças na ação dos voláteis durante o fracionamento, d) tipo de agente complexante responsável pelo transporte de Sn e W nos fluidos hidrotermais, e) taxa de interação entre fluido hidrotermal e rocha encaixante, e f) condições de temperatura, pressão, $\mathrm{pH}$, Eh e $f \mathrm{O}_{2}$ nos sítios deposicionais (Manning \& Henderson 1984, Jackson \& Helgeson 1985, Heinrich 1990, 
Marignac \& Cuney 1991, Taylor \& Wall 1992 e 1993).

Tanto cassiterita como wolframita nesses sistemas hidrotermais hospedam exsoluções de nióbio-tantalatos consideradas produtos do abaixamento da temperatura e/ou mudança no estado de oxidação do fluido hidrotermal coexistente. Experimentos laboratoriais associados a estudos de inclusões fluidas e isótopos estáveis, demonstram que cassiterita e wolframita são formadas a partir de fluidos hidrotermais salinos e que podem ocorrer em equilíbrio a temperaturas acima de $400^{\circ} \mathrm{C}$ (Kelly \& Rye 1979, Campbell et al. 1984, Ramboz et al. 1985, Stemprok 1990, Samson 1990, Heinrich 1990, Zhang et al. 1994). Nos greisens os principais fatores responsáveis pela precipitação de cassiterita e wolframita são o abaixamento da temperatura, pressão, $\mathrm{pH}$, Eh e $f \mathrm{O}_{2}$, fatores estes que estão, normalmente, relacionados à taxa de interação fluido hidrotermal-rocha encaixante, ao tipo de rocha encaixante e ao volume de interação entre fluidos de diferentes naturezas (Heinrich 1990, Taylor \& Wall 1993, Stemprok 1990, Marignac \& Cuney 1991).

No sistema plutônico Palenqueta a fase magmática responsável pela mineralização é atribuída ao albita granito, cuja evolução envolveu um eficiente mecanismo de fracionamento que culminou com a separação de uma fase hidrotermal rica em voláteis e elementos incompatíveis, a qual se deslocou para os níveis de ápice da intrusão ígnea e foi responsável pelas transformações tarde a pós-magmáticas que originaram os greisens (Souza \& Botelho 2002, Souza 2003, Souza et al. 2005)

Os cristais de cassiterita na zona de greisens do albita granito Palanqueta tendem a apresentar baixos conteúdos em Fe, Ti, $\mathrm{Nb}, \mathrm{Ta}, \mathrm{W}$ e conteúdos mais elevados de Sn. Tal característica é considerada como um dos fatores responsáveis pelo incomum e elevado grau de transparência desses cristais, permitindo inclusive a observação direta de seu conteúdo de inclusões fluidas (Souza 2003). Por outro lado, os cristais de wolframi- ta apresentam conteúdo em Fe mais elevado em relação ao $\mathrm{Mn}$, revelando franca migração para o membro ferberita $\left(\mathrm{Fe}_{0,52-0.86}-\mathrm{Mn}_{0,10-0,38}\right) \mathrm{WO}_{4}$, além de baixos conteúdos em Nb, Ta e Sn. Segundo Ivanova (1988), a composição da wolframita é uma função controlada pela razão $\mathrm{Fe} / \mathrm{Mn}$ na solução hidrotermal coexistente, pelo $\mathrm{pH}$ e pela $f \mathrm{O}_{2}$, independente do tipo de rocha encaixante, temperatura ou pressão. Polya (1988) expressa este equilíbrio entre a wolframita e a solução hidrotermal, negligenciando os íons traços, através da equação $\mathrm{FeWO}_{4 \text { (wolf.) }}+\mathrm{Mn}^{2+}{ }_{\text {(aq.) }} \leftrightarrow \mathrm{MnWO}_{4 \text { (wolf.) }}+\mathrm{Fe}^{2+}{ }_{\text {(aq.) }}{ }^{\circ}$

Considerando que o fracionamento isotópico é uma função direta da temperatura, a diferença isotópica observada entre cassiterita-wolframita-quartzo sugere que o abaixamento da temperatura no sistema hidrotermal, provavelmente devido à interação entre fluidos de diferentes naturezas, foi o fator mais importantes para a precipitação da cassiterita e wolframita nos greisens do albita granito Palanqueta. Nesse sentido, apesar da composição isotópica do fluido hidrotermal ter sido calculada indiretamente através de diferentes equações de fracionamento, os intervalos de variações isotópicas destes fluidos nos greisens $\left(\delta^{18} \mathrm{O} 4,26\right.$ a $7,33 \%$ ) mostram-se semelhantes, sugerindo que cassiterita e wolframita foram precipitadas em um mesmo intervalo de temperatura $\left(420^{\circ}\right.$ a $\left.460^{\circ} \mathrm{C}\right)$ e a partir do mesmo fluido hidrotermal de origem magmática.

Agradecimentos Os autores agradecem à Empresa Brasileira de Estanho S.A. (EBESA/Grupo Paranapanema) e à Companhia de Pesquisa e Recursos Minerais (CPRM-RO) pelo total apoio durante a etapa de campo. Ao Departamento de Engenharia de Minas da Universidade Federal de Minas Gerais pela concessão do uso do laboratório de microscopia eletrônica. Ao técnico Onésio Rodrigues Filho pela atenção e apoio durante as análises no laboratório de microssonda eletrônica da UnB.

\section{Referências}

Bettencourt J.S., Tosdal R.M., Leite Jr. W.R., Payolla, B.L. 1999. Mesoproterozoic rapakivi granites of the Rondônia Tin Province, southwestern border of the Amazonianian craton, Brazil - I. Reconnaissance U-Pb geochronology and regional implications. Precamb. Res., 95:41-67.

Bettencort J.S., Leite Jr., W.B., Goraieb C.L., Sparrenberger I., Bello R.M.S., Payolla B.L. 2005. Sn-polymetallic greisens-type deposits associated with late-stage rapakivi granites, Brazil: fluid inclusion and stable isotope characteristics. Lithos, 80:363-386.

Campbell A., Rye D.M., Peterson U. 1984. A hydrogen and oxygen isotope study of the San Cristobal mine, Peru: implications of the role of water to rock ratio for the genesis of wolframite deposits. Econ. Geol., 79:18181832.

Clayton R.N., O’Neil J.R., Mayeda T.K. 1972. Oxygen isotope exchange between quartz and water. Jour. Geoph. Res., 77(17):3057-3067.

Costi H.T., Horbe A.M.C., Borges R.M.K., Dall'Agnol
R., Rossi A., Sighnolfi G. 2000. Mineral chemistry of cassiterites from Pitinga Province, Amazonian Craton, Brazil. Rev. Bras. Geoc., 30(4):775-782.

Faure G. 1986. Principles of isotopic geology. $2^{\text {nd }}$, New York, John Wiley \& Sons, 589 p.

Giuliani G. 1987. La cassitérite zonée du gisement de Sokhret Allal (Granite des Zaër; Maroc Central): composition chimique et phases fluides associées. Mineral. Depos., 22(4):253-261.

Heinrich C.A. 1990. The chemistry of hydrotermal tin(tungsten) ore deposition. Econ. Geol., 85:457-481.

Hsu L.C. 1976. The stability relations of the wolframite series. Amer. Mineral., 61:944-955.

Ivanova G. 1988. Geochemical conditions of formation of various composition wolframites. Bull. Minieral., 111:97-103.

Jackson K.J. \& Helgeson H.C. 1985. Chemical and thermodynamic constraints on the hydrothermal transport and deposition of tin: I. Calculation of the solubility of 
cassiterite at high pressures and temperatures. Geoch. Cosmoch. Acta, 49:1-22.

Kelly W. \& Rye R.O. 1979. Geologic, fluid inclusion, and stable isotope studies of the tin-tungsten deposits of Panasqueira, Portugal. Econ. Geol., 74(8):1721-1822.

Kloosterman J.B. 1968. Uma província do tipo nigeriano no sul da Amazônia. Rev. Eng., Min. Metal., Rio de Janeiro, XLVII (278):59-64 e XLVII (280):167-168.

Leite Jr. W.B. 2002. A Suite Intrusiva Santa Clara (RO) e a mineralização primária polimetálica $(\mathrm{Sn}, W, \mathrm{Nb}, \mathrm{Ta}, \mathrm{Zn}$, $\mathrm{Cu}$ e $\mathrm{Pb}$ ) associada. Tese de Doutoramento, Instituto de Geociências, Universidade de São Paulo, 305 p.

Macavei J. \& Schultz H. 1993. The crystal structure of wolframite type tungstates at high pressure. Zeitsch. Kristallogr., 207:193-208.

Manning D.A. \& Henderson P. 1984. The behavior of tungsten in granitic melt-vapor systems. Contr. Mineral. Petrol., 86:286-293.

Marignac C. \& Cuney M. 1991. What is the meaning of granite specialization for $\mathrm{Sn}, \mathrm{W}$ deposit genesis? In: Pagel M.\& Leroy J.L. (eds.) Source, transport and deposition of metals. Balkema, Rotterdam, p. 771-774.

Möller P., Dulski P., Szacki W., Malow G., Riedel E. 1988. Substitution of tin in cassiterite by tantalum, niobium, tungsten, iron and manganese. Geoch. Cosmoch. Acta, 52:1497-1503.

Murciego A., Sanchez A.G., Dusausoy Y., Pozas J.M.M., Ruck R. 1997. Geochemistry and EPR of cassiterites from the Iberian Hercynian Massif. Mineral. Mag., 61:357-365.

Neiva A.M.R. 1996. Geochemistry of cassiterite and its inclusions and exsolutions products from tin and tungsten deposits in Portugal. Canad. Mineral., 34:745-768.

Polya D.A. 1988. Compositional variation in wolframites from the Barroca Grande mine, Portugal: evidence for fault-controlled ore formation. Mineral. Mag., 52:497503.

Priem H.N.A., Boelrijk N.A.I.M., Hebeda E.H., Verdurmen E.A.Th., Verschure R.H., Bom E.H. 1971. Granitic complexes and associated tin mineralizations of "Grenville"age in Rondônia, western Brazil. Geol. Soc. Amer. Bull., 82:1095-1102.

Ramboz C., Schnapper D., Dubessy J. 1985. The P-V-T-X-fO, evolution of $\mathrm{H}_{2} \mathrm{O}-\mathrm{CO}_{2}-\mathrm{CH}_{4}$ bearing fluid in a wolframite vein: reconstruction from fluid inclusion studies. Geoch. Cosmoch. Acta, 49:205-219.

Rollinson H. 1993. Using geochemical data: evaluation, presentation, interpretation. New York, Longman Scientific \& Technical, John Wiley \& Sons, 352 p.

Samson I.M. 1990. Fluid evolution and mineralization in a subvolcanic granite stock: the Mount Pleasant W-Mo-Sn deposits, New Brunswick, Canada. Econ. Geol., 85:145163.

Silva L.F.S., Costi H.T., Teixeira J.T. 1995. Faciologic mapping and preliminary petrography of Palanqueta albite granite - Bom Futuro, Rondônia State (Brazil). In: Symp. on Rapakivi Granites and Related Rocks. IGCPProject 315, Belém, Abstracts, p.73-74.

Silva L.F.S., Costi H.T., Dall'Agnol R., Teixeira J.T. 1997. Petrographic and geochemical characteristics of the tin-bearing albite granite of Morro da Palanqueta, Bom Futuro Mine, Rondônia, Brazil. In: Intern. Symp. on Granites and Assoc. Mineraliz. (ISGAM II), Salvador, Abstracts, p.155-156.

Souza V.S. \& Botelho N.F. 2002. Geologia do depósito de estanho do Bom Futuro (Rondônia) e composição dos fluidos nos sistemas de veios e greisens. In: Klein E.L., Vasquez M.L., Rosa-Costa L.T. (eds.) Contribuições à Geologia da Amazônia, 3,SBG-NO, p.199-214.

Souza V.S. 2003. Evolução magmática e modelo metalogenético do sistema vulcano-plutônico estanífero Bom Futuro (RO). Tese de Doutoramento, Instituto de Geociências, Universidade de Brasília, 240 p.

Souza V.S., Teixeira L.M., Botelho N.F. 2005. Datação $\mathrm{U}-\mathrm{Th}-\mathrm{Pb}$ de monazita hidrotermal e sua aplicação na geocronologia da mineralização de estanho em zonas de greisens do sistema granítico Palanqueta, depósito do Bom Futuro (RO). Rev. Bras. Geoc., 35(1):43-48

Stemprok M. 1990. Solubility of tin, tungsten and molybdenum oxides in felsic magmas. Mineral. Depos., 25:205-212.

Sparrenberger I. 2003. Evolução da mineralização primária estanífera associada ao maciço granítico Santa Bárbara, Rondônia. Tese de Doutoramento, Instituto de Geociências, Universidade de São Paulo, 254 p.

Taylor Jr. H.P. 1974. The application of oxygen and hydrogen isotope studies to problems of hydrothermal alteration and ore deposition. Econ. Geol., 69:843-883.

Taylor Jr. H.P. 1978. Oxygen and hydrogen isotope studies of plutonic granitic rocks. Earth Planet. Sci. Lett., 38:177210.

Taylor J.R. \& Wall V.J. 1992. The behavior of tin in granitoid magmas. Econ. Geol., 87:403-420.

Taylor J.R. \& Wall V.J. 1993. Cassiterite solubility, tin speciation, and transport in magmatic aqueous phase. Econ. Geol., 88:437-460.

Villanova M.T. \& Franke N.D. 1995. Serra do Bom Futuro - Rondônia: a volcanic-breccia pipe-hosted tin mineralization. In: Dall'Agnol R., Macambira M.J.B., Costi H.T. (eds.) Symp. on Rapakivi Granites and Related Rocks, IGCP-Project 315, Belém, Abstracts , p.83-84.

Waghorn J.G. 1974. The geology of Rondonia, western Brazil, with special reference to the tin-bearing granite complexes and placer deposits. PhD Thessis. Faculty of Science of the London, London, $239 \mathrm{p}$.

Waychunas G.A. 1991. Crystal chemistry of oxides and oxyhydroxides. In:. Lindsley D.H (eds.) Oxide Minerals: petrologic and magnetic significance. Mineralogical Society of America, Michigan, Reviews in Mineralogy, 25(2):11-68.

Zhang L-G, Liu J-X, Chen Z-S, Zhou H-B. 1994. Experimental investigations of oxigen isotope fractionation in cassiterite and wolframite. Econ. Geol., 89:150-157.

Zheng Y-F. 1992. Oxgen isotope fractionation in wolframite. Euro. Jour. Mineral., 4:1331-1335.

Manuscrito ID 14223

Submetido em 11 de maio de 2009 Aceito em 30 de novembro de 2009 